\title{
Tangence
}

\section{L'incipit : frontière et lieu stratégique de contact en littérature québécoise pour la jeunesse}

\section{Johanne Prud'homme}

Numéro 67, automne 2001

L'écriture pour la jeunesse : de la production à la réception

URI : https://id.erudit.org/iderudit/009616ar

DOI : https://doi.org/10.7202/009616ar

Aller au sommaire du numéro

\section{Éditeur(s)}

Presses de l'Université du Québec

ISSN

0226-9554 (imprimé)

1710-0305 (numérique)

Découvrir la revue

Citer cet article

Prud'homme, J. (2001). L'incipit : frontière et lieu stratégique de contact en littérature québécoise pour la jeunesse. Tangence, (67), 69-80.

https://doi.org/10.7202/009616ar
Résumé de l'article

Résumé

À titre de lieu de médiation entre le texte et son lecteur, entre le destinataire et le destinateur ou entre le narrateur et le narra- taire, l'incipit se révèle investi des marques de la relation qui unit l'auteur à son public. L'analyse de trois incipits exemplaires tirés d'oeuvres publiées au cours des vingt-cinq premières années d'existence de la littérature québécoise pour la jeunesse permet l'étude des rapports qui se nouent, au moment crucial de leur naissance, entre deux acteurs jusque-là absents du champ littéraire : l'auteur pour la jeunesse et son lecteur. L'étude de ces fragments textuels permet de déterminer quelles sont les straté- gies employées par le premier pour entrer en contact avec son public. Par ailleurs, l'analyse du discours incipitiel donne matière à dessiner, en filigrane, $Y$ ethos de l'auteur et le portrait de son jeune vis-à-vis. 


\title{
L'incipit : frontière et lieu stratégique de contact en littérature québécoise pour la jeunesse
}

\author{
Johanne Prud'homme, \\ Université du Québec à Trois-Rivières
}

Tout commencement est une prise de position.

Andrea Del Lungo

La frontière est un objet paradoxal. À son rôle de délimitation correspond une ambivalence fondamentale: elle met en contact ce qu'elle sépare'. Circónscrire, scinder ou mettre en relation, telles sont également, dans la perspective d'une poétique de la frontière, les fonctions limologiques ${ }^{2}$ pouvant être dévolues à l'incipit, cette frontière naturelle du texte sans laquelle ce dernier ne peut prétendre à l'existence. Le commencement, point cardinal de la lecture, est, comme l'affirme Friedrich D. E. Schleiermacher, "une annonce ou du moins une orientation générale "3. D'autres, après lui, n'hésiteront pas à considérer le début du texte comme l'atome primordial, le point d'origine de l'outure. L'une des propositions de Charles Grivel dans Production de lintérêt romanesque en fournit un exemple éloquent: "le roman n'est que le développement de son commencement [...] chaque élément constituant s'y rattache ${ }^{+}$. Tout entier tourné vers le texte qu'il inaugure, l'incipit conquiert, dans cette économie monadique, le statut de partie, certes, mais également celui de fragment porteur

1. Ce sont les géographes, surtout, qui ont mis en lumière cette ambivalence essentielle de la frontière. Voir, entre autres, les ouvrages suivants: JeanPierre Renard, Le gêgrapbe et les frontières, Paris, L'Harmattan, coll. "Les rendez-vous d'Archimède, 1997 et Michel Foucher, Fronts et frontieres. Un tour du monde géopolitique, Paris, Fayard, 1991.

2. De limologie, qui désigne la science du limen ("seuil "; fig. "début, commencement ").

3. Friedrich D. E. Schleiermacher, Herméneutique [1809], Lille, Éditions du Cerf, 1987, p. 102.

4. Charles Grivel, Production de l'intêrêt romanesque. Un état du texte (18701880), un essai de constitution de sa théorie, Paris, Mouton, 1973, p. 91. 
de tous les possibles. En comprendre les éléments constitutifs confère en quelque sorte au lecteur la possibilité de connaître le chiffre du texte et d'en permettre le décodage.

Si le lecteur savant trouve dans le décryptage des phrases liminaires de l'œuvre matière à circonscrire l'univers de la fiction - littéralement, "écrire autour" (circumscribere) —, qu'y trouve l'enfant lecteur, lui qui, naivement, quitte le confort douillet de son fauteuil ou la chaleur de l'épaule sur laquelle sa tête s'abandonne pour consentir à l'expérience troublante de l'entrée dans la terra incognita de l'œuvre? En faisant volontairement l'économie de la séduction tout adulte que nous inspirent les perspectives d'un Schleiermacher ou d'un Grivel, on ne peut manquer de percevoir l'incipit sous l'éclairage des relations transcendantes qu'il instaure avec le monde de l'“autre côté du miroir". À titre de lieu de médiation entre le texte et son lecteur, entre le destinataire et le destinateur ou entre le narrateur et le narrataire, le commencement se révèle, dès lors, être investi plus ou moins directement des marques de la relation qui unit l'auteur à son public. C'est de cette relation dont il sera question ici. L'analyse de trois incipits exemplaires, tirés d'œuvres publiées au cours des vingt-cinq premières années d'existence de la littérature québécoise pour la jeunesse, me permettra d'étudier les rapports qui se nouent, au moment crucial de leur naissance, entre deux acteurs jusque-là absents du champ littéraire: l'auteur pour la jeunesse et son lecteur. Quelles sont les stratégies employées par le premier pour entrer en contact avec son public? Qu'impliquent ces stratégies dans le cadre d'une production littéraire qui, fait inédit dans l'histoire de notre littérature, s'avère spécifiquement destinée aux enfants? En tentant de répondre à ces questions par une analyse du discours incipitiel, j'esquisserai en filigrane l'ethos de l'auteur et le portrait de son jeune vis-à-vis. Mais, tout d'abord, quelques mots sur l'histoire de ces derniers.

\section{Naissance de l'auteur québécois pour la jeunesse et de son public}

Quinze ans avant la publication de la première ceuvre pour la jeunesse, la littérature canadienne-française se trouve en pleine crise d'identité. Camille Roy, ardent défenseur d'une littérature essentiellement canadienne, s'insurge devant l'abandon qui la menace: "[l]a littérature canadienne existe, mais elle doit toujours 
lutter contre l'apathie asse grancie ciu puiviic, cumte lïnuifférence que l'on professe encore, même parmi nos gens instruits, pour cette chose légère et peu pratique qu'est la littérature ${ }^{5}$. Le désintérêt des élites, comme celui du public souvent jugé inculte, ne manque pas d'inquiéter les tenants d'une idéologie nationale qui voient dans le livre l'un des agents les plus efficaces pour le développement d'une conscience et d'une âme canadiennes. Cette défection du public adulte aura pour corollaire une modification subtile du discours propagandiste. Ainsi, une lecture des articles consacrés à la question de la littérature nationale, publiés entre 1910 et $1921^{\circ}$, permet d'observer l'émergence d'un discours apologétique exaltant les vertus d'une formation visant à développer le sens patriotique et les mérites d'une éducation intellectuelle de qualité pour les jeunes Canadiens français. Les raisons qui justifient cette mutation s'avèrent évidentes. Instruite, ayant "appris à voir et à sentir les choses autour d'elle" ", la jeunesse ne pourra que mieux apprécier les ouvres littéraires et se substituer à ce public "souffr[ant] d'une paresse intellectuelle routinière ${ }^{8}$ " qui, pis encore, est parfois atteint d'un "mal bien canadien [...] : la peur du livre" ". Il apparaît donc légitime de penser que c'est l'espoir mis dans cette jeunesse qu'on souhaite plus enthousiaste que ses aînés qui précipite, telle une chimie, l'avènement d'une littérature expressément conçue pour les enfants. Cette dernière, dont le but avoué est de renforcer le sentiment d'appartenance à la nation, croise donc d'emblée le champ de la politique et se révèle, à ses débuts, intimement liée au pouvoir et à la doxa. C'est sur ces

5. Camille Roy, ptre, "Tableau de lhistoire de lá littérature canadienne", L'enseignement primaire, Québec, n" 6, févrièr 1906, p. 338.

6. Voir, entre autres, les articles suivants (présentés par ordre chronologique): Camile Roy, ptre. "Tableau de I'histoire de la littérature canadienne ", art. cité, p. 336-342; Léo-P’atil Desrosiers, "Le point sensible ", La revtue nationale, Montréal, vol. 9. septembre 1919. p. 331-335; Fernand Rinfret, "La psychologie de notre mouvement littéraire ", Rez'ue trimestrielle canadienne, Montréal, n" 18,1919 , p. 170-177; Anonyme, "Nos livres, nos écrivains, notre jeunesse", La retue nationale, vol. 11, avril 1921, p. 29; Lbald Paquin, "Si nous avons une littérature ", La revue nationale, vol. 11, septembre 1921, p. 38-42; E.M., "Nos livres - Nos ecrivains", La revue nationale, vol. 11, novembre 1921, p. 104.

7. Léo-Paul Desrosiers, "Le point sensible", art. cité, p. 333.

8. Fernand Rinfret, "La psychologie de notre mouvement littéraire *, art. cité, p. 174 .

9. Fernand Rinfret, "La psychologie de notre mouvement littéraire", art. cité, p. 174 . 
prémisses que repose et s'impose la création du roman fondateur de la littérature québécoise pour la jeunesse - Les aventures de Perrine et de Charlot - dont on sait qu'il a été commandé à Marie-Claire Daveluy par la Société Saint-Jean-Baptiste de Montréal (SSJB) ${ }^{10}$. En ce début de siècle, alors qu'un discours convergent fait se rencontrer psychologie de l'enfant et pédagogie, la SSJB comprend sans peine qu'avant d'avoir accès aux "grandes" ceuvres de la littérature nationale, il est préférable, voire essentiel, que l'enfant fasse l'apprentissage de la lecture sous les auspices d'une littérature écrite à son unique intention. Paul-Émile Farley, dans le premier livre québécois posant un regard critique sur la littérature pour la jeunesse, résume bien l'esprit de l'époque:

[...] une littérature pour les enfants doit être écrite directement pour eux. S'il y a des principes généraux qui s'imposent partout, il y a une adaptation locale qu'un auteur na pas le droit de négliger. [...] Il nous appartient, à nous, Canadiens, d'accomplir cette ceuvre. (Euvre de dévouement et d'apostolat, mais ceuvre nécessaire. Les ouvrages qui donneront à notre jeunesse le goût des choses de l'esprit feront peut-être plus pour relever le niveau intellectuel de la race que les gros livres savants qu'on lit trop peu ${ }^{11}$.

Ces précisions historiques illustrent les tensions sous-jacentes à la création du "public jeunesse" québécois. $\mathrm{Si}$, dans un premier temps, on le perçoit comme lecteur à venir, mais potentiellement plus éclairé que ses devanciers, on lui reconnaît, à partir de 1923, le statut de lecteur réel, de public "certifié ". Cette transformation signe l'apparition de l'auteur québécois pour la jeunesse et de son public. Or, comme en témoignent nombre d'œuuves québécoises produites au cours de la première moitié du $x x^{e}$ siècle, c'est sur le seuil du texte, dans l'anticamera de la fiction, que se construisent graduellement, à la faveur de rencontres inaugurales réitérées, ces deux nouvelles figures. En toute innocence? On peut en douter. À titre de zone frontière entre le monde et le produit de sa recréation par l'écriture, l'incipit n'a de la pureté du premier souffle que l'apparence...

10. Publié tout d'abord sous forme de feuilleton dans les premières livrées (janvier 1921-mars 1922) de L'Oiseau bleu, organe jeunesse de la SSJB, le roman fera l'objet d'une publication en livre (Montréal, Bibljothèque de l'Action française, 1923).

11. Père Paul-Émile Farley, c.s.v, Livres d'enfunts, Montréal, Les Clercs de StViateur, 1929, p. 12-13. 


\section{L'incipit : une interface entre l'auteur et son public}

À l'évidence, la critique contemporaine reconnaît à l'incipit une fonction limologique de "mise en relation". Ainsi, les rapports au lecteur sont-ils déjà inscrits dans les fonctions qui, eu égard à une poétique du roman (pour la jeunesse ou non), échoient à l'incipit : "informer, intéresser et nouer le pacte de lecture ${ }^{12}$ ". Jean-Louis Morhange, intéressé essentiellement par les stratégies jouant un rôle de soutien au moment du franchissement du seuil, affirme fort pertinemment à leur sujet que, "loin de relever d'un simple jeu formel, [elles] semblent toucher au coeur du rapport des humains avec les fictions qu'ils produisent ${ }^{13}$ ". Pour leur part, Boie et Ferrer admettent, de manière beaucoup plus élusive, que "le début, en tant que bord, est aussi le point de contact avec le destinataire ${ }^{14_{n}}$. La signature jakobsonnienne se retrouve de même chez Del Lungo, qui voit dans le déchiffrement des phrases liminaires le "moment de contact entre le destinateur (l'auteur) et le destinataire (le lecteur) du texte ${ }^{15} "$; mais, plus important encore, c'est là, selon lui, que "se réalise un contact avec un lecteur réel, qui a matériellement ouvert le livre en commençant la lecture ${ }^{16}$.

Sur le limen textuel, au moment du décryptage des premières lignes de l'œuvre, le public — ce "monstre multicéphale et toujours inconnaissable ${ }^{17}$ n - se transforme donc en lecteur. En littérature pour la jeunesse, et ce d'autant plus qu'on la définit comme "intentionnelle", cette métamorphose fait de l'incipit un lieu stratégique de contact avec le lecteur visé par l'ouvre:

[c]ette image du lecteur [...] peut se dessiner dans les anticipations des normes et valeurs du public, dans les apostrophes du lecteur, $[\ldots]$ ainsi que dans de nouvelles disponibilités exigées

12. Vincent Jouve, La poêtique du roman, Paris, Sedes, coll. "Campus Lettres", 1997, p. 19.

13. Jean-Louis Morhange, "Incipits narratifs. L'entrée du lecteur dans l'univers de la fiction ", Poétique, Paris, n ${ }^{\circ} 104$, novembre 1995 , p. 387.

14. Bernhild Boie et Daniel Ferrer, "Les commencements du commencement ", Genèses du roman contemporain. Incipit et entrée en écriture, textes réunis par B. Boie et D. Ferrer, Paris, CNRS éditions, 1993, p. 34 ; c'est moi qui souligne.

15. Andrea Del Lungo, "Pour une poétique de l'incipit", Poétique, Paris, $\mathrm{n}^{\circ} 94$, avril 1993, p. 133.

16. Andrea Del Lungo, "Pour une poétique de l'incipit ", art. cité, p. 135.

17. Franc Schuerewegen, "Réflexions sur le narrataire. Quidam et Quilibet", Poétique, Paris, no 70 , avril 1987, p. 252. 
pour la réception. Dans le lecteur visé en tant que fiction de lecteur inhérente au texte apparaissent aussi des idées sur le rapport public-auteur. [...] Il est certain qu'un rapport existe entre la forme de présentation du texte et le lecteur auquel celui-ci est destiné ${ }^{1 \%}$.

De manière à déclarer leur intentionnalité et à afficher leur spécificité dans le champ littéraire, les romans fondateurs de la littérature québécoise pour la jeunesse s’ingénient à mettre en scène leur destinataire par le biais, principalement, d'apostrophes au lecteur. À cet égard, l'incipit se pose comme lieu privilégié de ces épiphanies et, en cette matière, fait figure d'espace public du texte. C'est sur le seuil, à la faveur de la "variation de [...] tension expositionnelle ${ }^{1 \%}$ "qu'implique l'acte de franchissement, que s'affirme l'identité singulière du lecteur. En cela, l'incipit confère ses limites identitaires au lecteur et, ce faisant, lui permet la distanciation et l'objectivation nécessaires pour pénétrer dans l'oeuvre. Cette distanciation, qui fait du discours incipitiel un "événement", pour reprendre le not de Paul Ricueur ${ }^{20}$, illustre une autre des fonctions limologiques de l'incipit, moins prégnante en littérature pour la jeunesse: celle de séparation.

Trois commencements exemplaires $" 1$ seront maintenant analysés, lesquels donnent lieu à autant de "programmes stratégiques. illustrant chacun les traits dominants de l'évolution d'une pratique qui a toujours cours dans les ceuvres d'aujourd'hui, celle de donner à voir, au seuil du texte, le sujet de l'énonciation et son interlocuteur.

18. Wolfgang Iser, Lacte de lecture. Thêrie de l'effet esthétique [1976], Liège, Mardaga, coll. "Philosophie et langage", p. 68). On doit le concept de lecteur "isé à Erwin Wolff ("Dor intendierte Leser ", Poetica, Munich, n" 4, 1971, p. 141 et suiv.).

19. Jean Samuel Bordreuil, "L'identité à l’épreveve de la ville. Élément pour unc géographie des (in) civilités's, dans Antoine Bailly \& Renato Scariati, Lbamanisme en géographie, Paris, Anthropos, coll. "Géographie - 1990, p. 49.

20. Paul Ricceur. Du texte à l'action. Fssais d'berméneutique II, Paris, Seuil, coll. "Points", 1986, p. 115.

21. Les incipits choisis sont extraits des ceuvres suivantes: abbé J.G. Gélinas, En veillant anec les petits de chez nous, Montréal, librairc Granger Frères Limitée, 1928; Lugène Achard, "La puce", Les contes du Kichelieu (1938), Montréal, Librairie générale canadienne, 1940; Maxine, Le marin de Saint-Malo. Jacques Cartier, Montréal, Beauchemin, coll. "Histoire du Canada pour les tout petits", 1946. Désormais, les références à ces ouvrages seront indiquées respectivement par les signes GEL, $A C H$ ou $M A X$, suivis de la page, et placécs entre parenthèses clans le corps du texte. 


\section{Apprendre à dire " nous »}

Nous avons été témoin des efforts de nos pères pour conserver le drapeau de la France au Canada; nous avons vu nos soldats épuisés, sans munitions, sans pain, écrasés par le nombre, capituler à Québec et à Montréal. Jamais un peuple ne pourra faire plus que nous avons fait pour la défense de la patrie.

Oui, mes enfants, nos pères consentirent à voir les couleurs d'Angleterre remplacer celles de la France. Mais c'était moyennant certaines conditions: le libre exercice de la religion catholique devait être sauvegardé, ainsi que les propriétés des Canadiens (GEL, p. 7).

Le "nous" qui inaugure les causeries historiques de l'abbé J. G. Gélinas peut aujourd'hui parâtre étrange. Or, à l'époque de la parution de l'ceuvre, cet embrayeur pronominal fort prisé par les nationalistes devient également l'un des outils privilégiés de la panoplie discursive de l'auteur pour la jeunesse. Ayant l'avantage de mettre en scène, et le peuple canadien, et le sujet de l'énonciation, le "nous" n'offre, cela dit, aucune assurance d'inclure le jeune lecteur: " "[n]ous" se dit d'une manière pour "moi + vous", et d'une autre pour "moi + eux" ${ }^{22}$ ". L'apparition, au deuxième paragraphe, d'une adresse au lecteur - "Oui, mes enfants" permettant symboliquement au destinataire d'éviter d'entrer en contact avec l'univers délétère des premières lignes, l'astreint du même coup à l'exclusion. Qui plus est, la séparation en paragraphes vient renforcer l'impression de césure. Cette stratégie a pour effet de poser au cour même de la zone frontière, une ligne de démarcation entre le monde de l'adulte et le monde de l'enfant. Ainsi, les premières lignes de l'incipit présentent-elles un discours à fonction testimoniale que vient alimenter en patbos la présence d'un champ notionnel relevant du désespoir. "Efforts de nos pères", "nos soldats épuisés", "sans munitions", "Sans pain", "écrasés par le nombre", autant de segments textuels qui mènent au verbe clef du paragraphe: "Capituler". Telle une réponse à une question posée in abstentia par les enfants, l'apostrophe explique les conséquences de la reddition. Le "mais" qui suit cette adresse est la pierre angulaire de la démonstration. Il permet d'énoncer les éléments qu'auront su préserver "nos pères" et, du même coup, instruit le lecteur. Pour ce faire, l'énonciateur

22. Émile Benveniste, Problèmes de linguistique générale, 1, Paris, Gallimard, coll. "Tel", 1983, p. 233. 
inscrit dans le discours incipitiel deux composantes incontournables de l'identité nationale canadienne-française: la religion catholique et la propriété des Canadiens ${ }^{23}$. Le procédé est habile puisque l'auteur, tout en affichant clairement sa posture idéologique, dote par la même occasion le lecteur des outils nécessaires pour la lecture des causeries historiques sur lesquelles ouvre l'incipit. Enfin, l'ensemble sémantique de cette deuxième partie de l'incipit se révèle on ne peut plus programmatique. Au champ sémantique du désespoir, s'oppose ici symétriquement celui de l'espoir qu'apporte malgré tout le compromis et que suggèrent les verbes "consentirent" et "remplacer". Ces derniers nous mènent en droite ligne vers le mot clef qui marque l'aboutissement du parcours: "sauvegarder". Comme sa position textuelle l'associe au monde de l'enfance, la sauvegarde de l'identité du peuple canadien-français apparait maintenant reposer entre les mains du jeune destinataire de l'œuvre. S'il s'acquitte comme il se doit de cette responsabilité, peut-être pourra-t-il, finalement, entrevoir la possibilité d'entrer dans le cercle de ceux qui, en toute légitimité, peuvent dire "nous".

\section{Découvrir la fragilité pour pouvoir vous parler}

Avec Michel Foucher, on peut constater, à la lumière de l'exemple précédent, que "les frontières sont du temps inscrit dans l'espace ${ }^{24}$. De fait, sous l'égide de l'auctoritas adulte pleinement investie des pouvoirs que lui confèrent le savoir et l'expérience, l'incipit, comme espace frontière, actualise le croisement entre l'ici-maintenant du lecteur et le continuum de l'Histoire que thématise le souvenir de nos "gloires nationales". Au cours des années 1930, toutefois, plusieurs auteurs expérimentent de nouvelles formes de prise de contact avec leur jeune public. Eugène Achard est de ceux-là. À preuve, le commencement de ses Contes du Richelieu:

Il y avait une fois... Rien que ce début vous annonce qu'il s'agit d'une histoire dans le genre de celles que l'on vous racontait, pour vous endormir, quand vous n'étiez pas plus haut que ça

23. En ce qui concerne la "propriété des Canadiens", il ne faut pas négliger le fait qu'au moment de la parution de l'ouvre, le mouvement coopératif, en plein essor, vise justement à préserver les intérêts et la propriété des Canadiens français.

24. Michel Foucher, Fronts et frontières, ouvr. cité, p. 43. 
[...]; une de ces histoires enfin comme seules les grand'mères savent en raconter et qui perdent toute leur saveur en passant par d'autres lèvres. Aussi vais-je laisser la parole à mémère et vous transcrire simplement son récit, tel que je l'ai entendu, tel du moins que je me le rappelle car, hélas, mémère n'est plus là pour m'aider; elle dort là-bas, dans le grand cimetière, à l'ombre de la croix, sous le grand orme en bordure de la haie, près du ruisseau qui murmure inlassablement aux morts ses de profundis mélancoliques, avant de les jeter au Richelieu, quelques arpents plus loin l...] ( $A C H$, p. 9).

Ici, on peut le constater, le dire et la parole ne s'inscrivent pas dans la temporalité linéaire d'un passé révolu légitimé par le discours de l'historien. Elles relèvent essentiellement de l'intemporalité du conte - familière à l'enfant - et du mouvement cyclique de l'éternel recommencement que permet la transmission des histoires. La position liminaire du "Il y avait une fois..." et la référence à cette "histoire du genre de celle que l'on vous racontait" instaurent, à cet égard, une relation intertextuelle, et donc transcendante, entre l'histoire racontée par Achard et toutes celles de la littérature internationale pour les enfants inspirées par la tradition orale. Si, par ce procédé référentiel, les Contes du Richelieu trouvent leur légitimation, ils n'en subvertissent pas moins la formule consacrée. De fait, une césure sémantique s'opère dès lors que le lecteur prend connaissance de l'histoire de mémère. Cette "fois" dont il est question n'est pas exclusivement celle, réitérée à l'infini, du traditionnel "Il était une fois". La formule désigne un événement singulier, une expérience qui ressortit à la mémoire possiblement défaillante - "tel du moins que je me le rappelle" - de l'énonciateur. Ce caractère lacunaire du souvenir individuel s'oppose à la véridicité et à l'incontestabilité de la mémoire collective toute patriarcale, représentée dans l'extrait précédent, et fait apparaitre une séparation dichotomique entre l'Histoire, unique, de "nos pères" $(G E L, 5)$ et les histoires de nos "grand'mères", entre l'écriture et l'oralité. En inscrivant son œuvre dans certe portion du champ littéraire dite "pour la jeunesse", Eugène Achard marque sa filiation, non pas aux écrivains de son sexe, mais bien aux femmes qui, comme le souligne Camille Roy, "ont donné en ce genre les œuvres les plus nombreuses et les meilleures ${ }^{25}$ ".

25. $\mathrm{M}^{\mathrm{kr}}$ Camille Roy, Manuel d'bistoire de la littérature canadienne de langue française, Montréal, Beauchemin, 1942, p. 161. 
Par ailleurs, dans cet incipit que teinte une affectivité certaine, Achard donne à voir un auteur fragile, conscient de la précarité de toute transmission et de l'instabilité des processus mémoriels. De cette manière, il met en abyme l'expérience lacunaire du jeune enfant et oppose aux modèles unilatéraux de prise de contact entre l'auteur et son public, une configuration relationnelle qui se distingue par la réciprocité qu'elle induit entre ces deux acteurs ${ }^{26}$. Une telle stratégie tend à dématérialiser les frontières entre le monde de l'adulte et celui de l'enfant, sans pour autant déposséder ce dernier des cadres nécessaires à sa tranquillité. Lorsque Eugène Achard aborde la question de la mort dans les phrases liminaires des Contes du Richelieu, c'est pour mieux signifier l'importance de la fiction dans la vie et l'importance de la vie dans la fiction, affirmant de cette façon la perméabilité de la frontière entre la vie et la mort. Mémère, qui fait vivre par ses histoires le murmure inlassable du ruisseau menant au Richelieu, ne s'est "endormie", finalement, que pour rejoindre les lieux de ses fictions.

\section{Vous montrer le chemin}

Les cas précédents sont exemplaires en ce qu'ils illustrent bien les deux grandes tendances qui ont cours au début du siècle dès lors qu'à l'incipit l'auteur s'adresse à son lecteur. Si l'on s'en tient à sa thématique, l'incipit suivant est, lui aussi, le fruit de son époque. Cela dit, il répond, avant la lettre, à ce précepte moderne qui veut que "l'accessibilité du texte conditionne [...] toute lecture juvénile ${ }^{27}$ ".

Ce beau pays que vous habitez, heureux petits enfants canadiens, avez-vous jamais songé à son histoire? Désirez-vous la connaître? Oui, sans doute.

Alors suivez bien les images qui illustrent ce récit et vous allez y trouver un beau conte - un conte vrai et captivant - concernant notre cher Canada.

26. Ce modèle, utilisé et repris par les auteurs contemporains d'Eugène Achard, fait encore force de loi aujourd'hui, à la différence près que l'adulte mis en scène par les écrivains d'alors a été remplacé, le plus souvent, par un narrateur enfant.

27. Ganna Ottevaere-van Praag, Le roman pour la jeunesse. Approches, définitions, techniques narratives, Berne et New York, Peter Lang, 1997. p. 9. 
Voyez, d'abord, cette ville bretonne d'une ancienne province française : c'est Saint-Malo avec son beau port de mer. Un bambin de cinq ans, tenant la main de son papa, circule au bord de la mer, parmi les quais et les passerelles. [...] Cet enfant se nomme Jacques -- Jacques Cartier (MAX, p. 5).

Le liminaire du Marin de Saint-Malo affiche, de toute évidence, une volonté d'entrer en contact avec le lecteur, mais également celle, plus pédagogique, de l'orienter dans sa lecture. Tout, dans cet incipit, concourt à l'élaboration d'une symétrie parfaite entre le contenu du texte et l'acte de la lecture. Les interrogations, leur réponse, les indications relevant d'une praxis de lecture - "alors suivez bien les images", "voyez, d'abord" - la présentation d'un Jacques Cartier enfant auquel le lecteur peut s'identifier, voilà autant d'éléments qui encadrent, en le contraignant, l'enfant lecteur. Même la question nationale est présentée de manière pragmatique par l'évocation spatiale, plus concrète pour ces "heureux petits enfants canadiens", du "pays où vous habitez", de "ce cher Canada". L'Histoire, elle, devient histoire, "conte vrai". Aucun blanc, aucun espace à combler: ce texte surdétermine son intentionnalité. L'auteur ne se sera jamais autant intéressé au lecteur qui, lui, n'auráa jamais paru aussi démuni et aussi peu autonome.

L'œuvre de Maxine, originale pour son époque, le serait bien moins aujourd'hui. Bien que l'énonciatrice se pose ici comme adulte, ce qui est rarement le cas dans les ceuvres contemporaines pour la jeunesse qui préfèrent plutôt mettre en scène un jeune narrateur homodiégétique, les procédés qu'elle utilise ressemblent fort à ceux que privilégie la littérature actuelle. Cette manière de prendre le lecteur par la main pour tenter de lui proposer une expérience entièrement adaptée à ce qu'il est, sait et pense me semble périlleuse. Si le jeune destinataire des cuvres pour la jeunesse a besoin, pour comprendre l'keuvre, d'y retrouver des éléments connus, il a également soif de l'altérité qu'elle peut lui apporter. Tout incipit ouvre sur un ailleurs, mais il est des ailleurs si spéculaires qu'on en arrive à douter d'avoir traversé la frontière...

Pierre Bourdieu, dans Les règles de l'art, évoque l'acte de Regere fines qui "consiste à tracer en lignes droites les frontières, 
80

à séparer "l'intérieur de l'extérieur" ${ }^{28}$ ". Nous avons vu qu'en déterminant la lisière de l'incipit, l'auteur, comme régisseur de l'espace textuel, définit aussi les conditions de la séparation entre le monde du quotidien et le monde du texte. Cette vision de l'incipit comme ligne n'est cependant pas suffisante pour comprendre toute la portée du texte liminaire qui requiert, pour se manifester comme zone, un double tracé. Andrea Del Lungo n'ignore pas ce fait lorsqu'il définit l'incipit comme "un fragment textuel qui commence au seuil d'entrée dans la fiction [...] et qui se termine à la première fracture importante du texte ${ }^{29}$. Dans les œuvres de la littérature dite générale, la présence visible du sujet énonciateur ne conduit pas nécessairement à une compartimentation de l'espace incipitiel. Par contre, dans les premières œuvres québécoises pour la jeunesse, l'espace compris entre le seuil et la fracture apparaît le plus souvent cloisonné, tel un vestibule qu'il faut franchir avant d'avoir accès à la fiction. Or, c'est précisément cet "arrêt", au sens littéral du terme puisqu'il retarde l'entrée dans le corps du texte, qui met en lumière la fonction limologique de "mise en relation" de la zone incipitielle en tant que lieu de prise de contact du lecteur avec le monde du texte et de l'auteur avec son public.

28. Pierre Bourdieu, Les règles de l'art [1992], Paris, Seuil, coll. "Points", 1998, p. 283.

29. Andrea Del Lungo, "Pour une poétique de l'incipit", art. cité, p. 137. 